\title{
The Effect of Training Program to Improve Fine Motor Reaction Speed in Light of Kinematic Characteristics of Half Roundhouse Kick (Mawashi-Geri Jodan) Within Karate Players.
}

\section{Dr. Emad Abdel Fattah Alserssi}

Assistance professor, Sports Training Department, Faculty of Physical Education, Tanta University. Egypt.

\section{Abstract}

This study aim to identify Kinematic characteristics of the half roundhouse kick (Mawashigeri Jodan) and Identify the effect of training program to improve fine motor reaction speed in light of kinematic characteristics of half roundhouse kick (Mawashi-geri Jodan)

The researcher used the descriptive approach to identify Kinematic characteristics of Mawashi-geri Jodan skill and experimental approach with one group design for application of proposed program for development of fine motor reaction speed for skill under discussion.

The research sample consists of (10) first-class players from Almonofiyah Karate region, also one international player selected as sample of kinematic analysis for skill under study

The most important results:

- Knee and pelvis joints represent great importance in Mawashi-geri kick for karate players

- Body's center of gravity plays a great role in success of performing motor requirement of Mawashi-geri for karate players.

- It is important to use exercises similar to skill performance

The researcher recommended using kinematic determinants derived from this research when developing speed training program and associate with other physical abilities like accuracy. .

\section{Introduction:}

tudy biomechanics indicators is
considered from basic research in studying movements and identifying factors affecting them, which led formation of foundation to understand complex aspects of human movements; so the trend in recent research in sports filed is to examine biomechanics indicators for some motor skills and development its quantitative and objectivity criteria aiming to raise performance skill level. (Jamal Mohammed Aladdin, 1994: p12)
Essam Abdel-Khalek (1991, p92), Charles, Ruth (1994, p159) emphasized on necessity of physical fitness exercises arising from skill performance nature of sport activity itself; where training through specific exercise is a way to adapt individual possibility to perform motor duty required, so physical characteristics development programs should basically be based on type and nature of skills performance, within each stage art performance, through specific exercise of where its movements in composition, requirements and work direction are similar to those movements 
performed during the competition and that to raise technical performance level for technical stages of performance.

Talha Hossam El Din (1998, p48) argue that privacy is of the most important principles of sports training as it is the best way for developing performance through training in a manner similar to large degree with the method of skill itself, and the more privacy training the more training outcome through training process.

Sharif Al-Awadi (1998, 13), mention that it has become clear that information about skill performance characteristics should be available to the coach in order to facilitate training on it through detection of the interrelationships between body parts while performing the skill that cannot be obtained, without follow-up and analysis of player movement through skill performance stages. "Stanndler" confirmed that identifying performance characteristics requires researchers knowledge of all information associated with work of body parts, joints and muscles so that they can discuss performance analysis under study, especially if the goal is to access information communicate it with both practitioner and beginner to achieve ideal performance.

Sawsan Abdul Moneim(1997, p37) argue that the interest in movement study requires familiarity of rules of motor analysis that rely on basic principles for each of kinesiology, anatomy, biomechanics, and other sciences associated with skill and technical information on any skill and its performance method in a form of information collection that help to determine the compound actions required to achieve this performance with highest possible efficiency and with minimal effort.
Verkhechchnesky (1993, p265) see that skill improvements effectiveness is mainly related to the harmonization process to master performance art with physical abilities training and athletes' physical preparation methods.

Ahmed Mahmoud Ibrahim al (1999, p27) emphasizes that Karate sport, like other sports- recently- have been subject to many of scientific developments in various aspects of players, especially in their training programs which lead to change attack and defense methods.

Ahmed Mahmoud Ibrahim (1991, pp50-51) mention that kicking is one of the mainstays of karate structure. In this regard, Wageh Shamandi(2002, p95) adds that winning in kumite "kumite" requires many factors, including proficiency in many kicks, skill performance will not will not be executed if using kicks strongly and effectively is neglected ; where motor kicks group play an important and key role in the attack due to its using of large muscle groups which characterizing legs for production of great strength and efficient speed to employ them in skill and tactical performance.

Imad Abdel Fattah Alserssi (2001, p44) via Nakayama (1983) indicates that "Mawashi Geri" kick is one of the strongest attack skills and the most commonly used in karate, but it needs time and effort in upgrading through training; this kick difficulty appear in player need for balance ability because the player is based on one foot, as well as its performance through different distances (short - medium long) and then followed by return movement after reaching the target with control of the resulting force not to cause injury to opponent or causing breach to player's balance. 
Sharif Al Awadhi, Omar Mohammed Labib (2004, p211) indicates that a successful attack is done by full control from player, which is heading towards a specific gap in the opponent's body accurately and quickly in the direction of well-known and well studied shooting area which is suitable for type of skill used in the attack.

Researcher believes - as international referee - and due to his presence in most local and international tournaments that player's mastering all kicks generally, and Mawashigeri especially, is crucial in obtaining a score of 3 full points (Ippon) in case of performance in (Jodan) area, or two full points (Waza-ari) in case of performance in (Chudan) area or raising player's performance level in feet movements or trying to get in and out of competitor field or trying to perform deceive with legs to attack with arm, all that have given great importance to try to understand, analyze and raise Mawashi-geri performance level, according to the latest amendments in the law of Kumite competitions:

- Player gets three points when perform it in the Jodan.

- Player gets two points when perform it in the Chudan.

- Considered preliminary stage for most other kicks.

- Enable player to win the match quickly and save his effort other matches if performed successfully for three times. (Imad Abdel Fattah Alserssi, 2011:pp 22-26)

Researcher believes that the attempt to identify kinematic characteristics of this skill as one of the important determinants and final outer appearance; which lies in clarifying its performance requirements, may contribute to facilitate of designing appropriate development methods and this is what push the researcher to try to identify the most important indicators and characteristics of Mawashi-geri kick and try to employ them to develop exercises to enhance fine motor reaction speed, where skill performance necessarily associated with reaction and accurate elements to complete it and get the desired goal of the performance which is scoring "3 points". Skills performance has no importance without motor reaction or performance accuracy needed to score the 3 points. A combination of skill performance, motor reaction motor and shooting accuracy in the right place and in the right time is the real objective and of training process. This prompting the researcher to study the kinematic characteristics of Mawashi-geri Jodan kick to improve the fine motor reaction speed for Karate players

\section{Research objectives:}

1- Identifying Kinematic characteristics of the half roundhouse kick (Mawashi-geri Jodan)

2- Identify the effect of training program to improve fine motor reaction speed in light of kinematic characteristics of half roundhouse kick (Mawashi-geri Jodan)

\section{Research queries:}

1- What are the most important Kinematic characteristics of the half roundhouse kick (Mawashi-geri Jodan)?

2- What is the effect of training program to improve fine motor reaction speed in light of kinematic characteristics of half roundhouse kick (Mawashi-geri Jodan)? 


\section{Research Terms:}

Fine motor Reaction speed: is the karate player's ability to respond to certain stimuli in the least possible time with the performance of a certain skill in certain place with particular purpose. (Procedural definition).

Ippon: three points awarded to the player in case of kicking in Jodan area (face - head neck), vacuuming or withdraw or throw the player for the other player to drop him on the ground and follow with right (punch- kicking a. (Procedural definition)

Jodan: a shooting area (face - head - neck). (Procedural definition)

Waz-ari: two points awarded to the player in case of kicking in Chudan (chest - abdomen sides). (Procedural definition).
Chudan :a shooting area: (chest - abdomen sides - shoulders - pubic bone). (Procedural definition)

\section{Research procedures:}

\section{Methodology:}

The researcher used the descriptive approach to identify Kinematic characteristics of Mawashi-geri Jodan skill and experimental approach with one group design for application of proposed program for development of fine motor reaction speed for skill under discussion.

\section{Research Society and sample:}

\section{A - Descriptive Sample:}

The descriptive sample included one player from the Egyptian kumite team (Ahmed Nasr) who is one of the most players using Mawashi-geri Jodan

Table (1)

Descriptive sample specification

\begin{tabular}{|c|c|c|c|c|c|c|c|}
\hline 莺 & 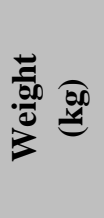 & 总 & 莺 & 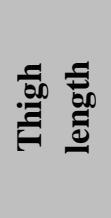 & 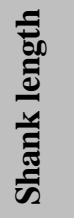 & 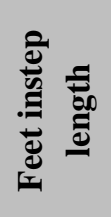 & $\begin{array}{l}\text { Champions won } \\
\text { by layer }\end{array}$ \\
\hline 172 & 67 & $\begin{array}{c}12 \\
\text { years }\end{array}$ & $\begin{array}{l}107 \\
\text { cm }\end{array}$ & $\begin{array}{l}\mathbf{5 7} \\
\mathbf{c m}\end{array}$ & $\begin{array}{l}\mathbf{5 0} \\
\mathbf{c m}\end{array}$ & $\begin{array}{l}30 \\
\text { cm }\end{array}$ & $\begin{array}{c}\text { Arab Champion - African champions - } \\
\text { Mediterranean - World Cup }\end{array}$ \\
\hline
\end{tabular}

\section{B. Experimental research sample:}

The research sample consists of (10) first-class players from Almonofiyah Karate region out of (30) player society. 
Table (2)

Statistical description of the experimental sample $\mathbf{n}=\mathbf{1 0}$

\begin{tabular}{|l|c|c|c|c|c|c|c|}
\hline No. & Variables & $\begin{array}{c}\text { Measurement } \\
\text { units }\end{array}$ & Mean & Median & $\begin{array}{c}\text { Standard } \\
\text { Deviation }\end{array}$ & Kurtosis & Skewness \\
\hline 1. & Age & Year & 19.7 & 19 & 2.31 & -1.03 & -1.22 \\
\hline 2. & Training Age & Year & 5.7 & 5.5 & 2.54 & 0.04 & -1.23 \\
\hline 3. & Weight & Kg. & 59.7 & 60 & 2.31 & -0.82 & -0.93 \\
\hline 4. & Height & Cm & 162.3 & 162 & 2.54 & -0.29 & 1.26 \\
\hline 5. & Leg length & Cm & 104.5 & 107 & 6.22 & -1.75 & 1.84 \\
\hline 6. & Thigh length & Cm & 50.1 & 50 & 0.99 & 0.61 & 0.15 \\
\hline 7. & Shank length & Cm & 45.6 & 46 & 1.5 & 1.58 & 2.3 \\
\hline 8. & Foot length & Cm & 29.9 & 30 & 1.19 & 0.23 & 0.36 \\
\hline 9. & Pelvis & Cm & 3.6 & 3.5 & 0.96 & 0.11 & 0.62 \\
\hline 10. & Reaction speed & Second & 3.47 & 3.5 & 1.66 & 0.6 & -0.05 \\
\hline 11. & Accuracy & Mark & 2.53 & 3 & 1.6 & 1.05 & 0.88 \\
\hline 12. & $\begin{array}{l}\text { Fine accurate } \\
\text { reaction speed }\end{array}$ & Second & 2.9 & 3 & 1.7 & 1.33 & 0.176 \\
\hline
\end{tabular}

Table (2) demonstrate arithmetic mean, median and standard deviation, kurtosis and skewness coefficient of the experimental group in age, training age, anthropometric measurements and physical variables under discussion and it is clear that all skewness values range between $( \pm 3)$, which declare the normal distribution of the variables.

\section{Data collection tools:}

1. Kinetic analysis Win Analyze program.

2. Brochure for recording players data (sample).

3. Control and guidance signs.

4. Rubber (different resistors).

5. Restameter to measure lengths and weights.

6. Kick rackets for training.

7. Kick rackets to performance accuracy.
8. Televised Camera.

9. VCR.

10. A device for measuring Fine accurate reaction speed time (designed by the researcher).

11. Wall Ladder.

12. Swedish seats.

Measurements and tests used in Experimental Research:

1. Weight measurement.

2. Height measurement.

3. Leg length.

4 Shank length.

5. Thigh length

6. foot length of.

B - Physical skill tests: 
1. Test to measuring motor reaction speed of Mawashi-geri skill.

2. Test to measure performance accuracy of Mawashi-geri skill.

3. Test to measure Fine accurate reaction speed time of Mawashi-geri skill.

\section{Pilot studies :}

First pilot study carried out on the period 3/1/2012- 08/01/2012, at Faculty of Physical Education - Tanta University - and aimed to:
- Determining the appropriate vediograph place where the player is being able to move free to perform Mawashi-geri skill.

- Fix Cameras places in front, side, and transverse levels (3 cameras) threedimensional imaging.

Second pilot study carried out on the period aimed to ensure validity and reliability of tests used.

Table (4)

Tests validity (motor reaction speed - accuracy - fine motor reaction speed) tests

\begin{tabular}{|c|c|c|c|c|c|c|}
\hline \multirow{2}{*}{ Variables } & \multicolumn{2}{|c|}{ Distinctive group } & \multicolumn{2}{|c|}{ Un-distinctive group } & \multirow{2}{*}{$\begin{array}{c}\text { Mean } \\
\text { differences }\end{array}$} & \multirow{2}{*}{$\mathbf{T}$} \\
\hline & Mean & SD & Mean & SD & & \\
\hline motor reaction speed & 1.41 & 7.04 & 1.11 & 4.62 & 0.3 & $11.41 *$ \\
\hline Accuracy & 2.8 & 0.63 & 0.7 & 0.48 & 2.1 & $8.35 *$ \\
\hline Fine motor reaction speed & 1.43 & 6.38 & 1.15 & 4.55 & 0.28 & 11.38* \\
\hline
\end{tabular}

T significance at 0.05 level $=2.26$

Table (4) demonstrate results of validity for lowest value while the $\mathrm{T}$ significance value at tests under discussion where $\mathrm{T}$ value in Fine $0.05=2.26$ This confirm validity of tests motor reaction speed was "11.41" as the used. highest value and in accuracy test " $8.35 "$ as

Table (5)

Tests reliability (motor reaction speed - accuracy - fine motor reaction speed)

\begin{tabular}{|c|c|c|c|c|c|}
\hline \multirow{2}{*}{ Variables } & \multicolumn{2}{|c|}{ Distinctive group } & \multicolumn{2}{|c|}{ Un-distinctive group } & \multirow{2}{*}{$\begin{array}{c}\text { Mean } \\
\text { differences }\end{array}$} \\
\cline { 2 - 5 } & Mean & SD & Mean & SD & 0.89 \\
\hline Motor reaction speed & 1.41 & 7.04 & 1.43 & 5.67 & 0.8 \\
\hline Accuracy & 1.4 & 1.17 & 1.8 & 1.03 & 0.99 \\
\hline Fine motor reaction speed & 1.43 & 6.38 & 1.43 & 5.79 & 0.06 \\
\hline
\end{tabular}

Significance limit at 0.05 level $=\mathbf{0 . 3 6 4}$

Table (5) results reveal reliability of test Pre-measurement: used. 
Pre- measurement was conducted on the experimental research sample in the period from $10 / 01 / 2012$ to $12 / 01 / 2012$ at covered hall, Shebin El Koum stadium - Monofia.

\section{Training program planning:}

Researcher has developed a training program in the light of results of Kinematic characteristics of Mawashi-Geri skill where performance stages were divided into five stages (standby position - preliminary stage first major phase - second major phase returning to standby position ).
Total no of cadres (50) have been distributed to the five stages. It was taken into account that exercises should be in the form similar to skill performance in each of the five stages in light of training loads directed to development of motor reaction with, audiovisual stimuli, with different goals and training on it to develop accuracy, then merge the two elements to improve the performance level of fine motor reaction speed fine for (Mawashi-gerry) skill.

Table (6)

Elements of the proposed training program

\begin{tabular}{|c|c|c|}
\hline No. & Program Elements & Experts opinions \\
\hline 1- & Program implementation time & 6 weeks \\
\hline $2-$ & Weekly training sessions & 4 units \\
\hline 3- & Training session times & 60 minutes \\
\hline $4-$ & Total number of training sessions & 24 training unit \\
\hline $5-$ & Total program time & 1440minutes \\
\hline 6- & Used training Loads & Medium - high - Maximum \\
\hline $7-$ & Training method used & high intensity interval \\
\hline \multirow{3}{*}{ 8- } & \multirow{3}{*}{ Tests used } & $\begin{array}{l}1 \text { - Motor reaction speed tests } \\
\text { (designed by the researcher) }\end{array}$ \\
\hline & & $\begin{array}{l}2 \text { - Performance accuracy test } \\
\text { (designed by the researcher) }\end{array}$ \\
\hline & & $\begin{array}{l}3 \text { - Fine motor reaction speed tests } \\
\text { (designed by the researcher) }\end{array}$ \\
\hline
\end{tabular}

The program has been applied during the period from 14/1/2012 until 27/2/2012, it was taking into account what referred to by both Mohamed Hassan Allawi and Abu-Ela Abdel Fattah (1998, pp164-165) and Mohammad Hassan Allawi (1990, pp138141) that attention must be paid to development of muscle strength with what suit requirements of specialized sport as muscle strength is an important key factor to ensure development of motor speed and thus speed of motor reaction.
Researcher considered training using light resistors represented is in $1,1 \frac{1 / 2}{2}, 2 \mathrm{~kg}$ sand bags, rapped on thigh or shank during exercises which is similar to skill performance and also the use of real rubber resistant which do not impede motor skill path, taking into account that all exercises are associated with stimuli given by coach (whether audio or light) to improve players' response. 
Table (7)

Total program timetable distribution on the contents (1440 minutes)

(Warm-up - motor reaction speed - accuracy - fine motor reaction speed- final-part)

\begin{tabular}{|c|c|c|c|c|}
\hline No. & Content & $\%$ & Time & Time per unit \\
\hline 1. & Warm-up & $15 \%$ & 216 minutes & 9 minutes \\
\hline 2. & Motor reaction speed & $20 \%$ & 288 minutes & 12 minutes \\
\hline 3. & Accuracy & $20 \%$ & 288 minutes & 12 minutes \\
\hline 4. & Fine motor reaction speed & $35 \%$ & 504 minutes & 21 minutes \\
\hline 5. & Final-part & $10 \%$ & 144 minutes & 6 minutes \\
\hline 6. & Total time & $100 \%$ & 1440 minutes & 60 minutes \\
\hline
\end{tabular}

Table (8)

Proposed training program for 6 weeks

\begin{tabular}{|c|c|c|c|c|c|c|c|}
\hline Week & Unit Parts & Time & Content & Intensity & Repetition & Rest & Sets \\
\hline \multirow{5}{*}{$\begin{array}{c}1^{\text {st }} \\
\text { and } \\
2^{\text {nd }}\end{array}$} & Warm-up & $\begin{array}{l}9 \text { minutes } x \\
8 \text { units }\end{array}$ & Exercises (1-7) & $50 \%$ & 1 & without & 1 \\
\hline & $\begin{array}{c}\text { Motor reaction } \\
\text { speed }\end{array}$ & $\begin{array}{l}12 \text { minutes } x \\
8 \text { units }\end{array}$ & Exercises (19-25) & $70 \%$ & 10 times & $\begin{array}{c}120 \\
\text { second }\end{array}$ & 3 \\
\hline & Accuracy & $\begin{array}{l}12 \text { minutes } x \\
8 \text { units }\end{array}$ & Exercises $(33,24,35)$ & $70 \%$ & 10 times & $\begin{array}{c}120 \\
\text { second }\end{array}$ & 3 \\
\hline & $\begin{array}{l}\text { Fine motor } \\
\text { reaction speed }\end{array}$ & $\begin{array}{l}21 \text { minutes } x \\
8 \text { units }\end{array}$ & $\begin{array}{c}\text { Exercises }(23,24, \\
25),(23,24,35)\end{array}$ & $70 \%$ & 6 times & $\begin{array}{c}120 \\
\text { second }\end{array}$ & 1 \\
\hline & Final-part & $\begin{array}{l}6 \text { minutes } x \\
8 \text { units }\end{array}$ & $\begin{array}{c}\text { Cool-down and } \\
\text { swinging exercises }\end{array}$ & $30 \%$ & 1 & $\begin{array}{c}120 \\
\text { second }\end{array}$ & 1 \\
\hline \multirow{5}{*}{$\begin{array}{l}3^{\text {rd }} \\
\text { and } \\
4^{\text {th }}\end{array}$} & Warm-up & $\begin{array}{l}9 \text { minutes } x \\
8 \text { units }\end{array}$ & Exercises (1-7) & $60 \%$ & 1 & without & 1 \\
\hline & $\begin{array}{c}\text { Motor reaction } \\
\text { speed }\end{array}$ & $\begin{array}{c}12 \text { minutes } x \\
8 \text { units } \\
\end{array}$ & Exercises (26-32) & $90 \%$ & 10 times & without & 3 \\
\hline & Accuracy & $\begin{array}{l}12 \text { minutes } x \\
8 \text { units }\end{array}$ & Exercises $(35,36,37)$ & $80 \%$ & 10 times & without & 3 \\
\hline & $\begin{array}{c}\text { Fine motor } \\
\text { reaction speed }\end{array}$ & $\begin{array}{l}21 \text { minutes } x \\
8 \text { units }\end{array}$ & $\begin{array}{c}\text { Exercises (26-32), } \\
(35,37)\end{array}$ & $80 \%$ & 6 times & $\begin{array}{c}120 \\
\text { second }\end{array}$ & 3 \\
\hline & Final-part & $\begin{array}{l}6 \text { minutes } x \\
8 \text { units }\end{array}$ & $\begin{array}{c}\text { Cool-down and } \\
\text { swinging exercises }\end{array}$ & $30 \%$ & 1 & $\begin{array}{c}120 \\
\text { second }\end{array}$ & 1 \\
\hline \multirow{5}{*}{$\begin{array}{c}5^{\text {th }} \\
\text { And } \\
6^{\text {th }}\end{array}$} & Warm-up & $\begin{array}{l}9 \text { minutes } x \\
8 \text { units }\end{array}$ & Exercises (1-7) & $70 \%$ & 1 & $\begin{array}{c}120 \\
\text { second }\end{array}$ & 1 \\
\hline & $\begin{array}{c}\text { Motor reaction } \\
\text { speed }\end{array}$ & $\begin{array}{l}12 \text { minutes } x \\
8 \text { units } \\
\end{array}$ & $\begin{array}{c}\text { Skill fight } \\
\text { performance }\end{array}$ & $\begin{array}{c}90- \\
100 \% \\
\end{array}$ & $18-12$ & $\begin{array}{c}120 \\
\text { second }\end{array}$ & 3 \\
\hline & Accuracy & $\begin{array}{l}12 \text { minutes } x \\
8 \text { units } \\
\end{array}$ & $\begin{array}{c}\text { Skill fight } \\
\text { performance }(35-37)\end{array}$ & $\begin{array}{c}90- \\
100 \% \\
\end{array}$ & $18-12$ & without & 3 \\
\hline & $\begin{array}{l}\text { Fine motor } \\
\text { reaction speed }\end{array}$ & $\begin{array}{l}21 \text { minutes } x \\
8 \text { units }\end{array}$ & $\begin{array}{c}\text { Skill fight } \\
\text { performance } \\
(25,35,37)\end{array}$ & $\begin{array}{c}90- \\
100 \%\end{array}$ & $6-10$ & without & 3 \\
\hline & Final-part & $\begin{array}{l}6 \text { minutes } x \\
8 \text { units }\end{array}$ & $\begin{array}{c}\text { Cool-down and } \\
\text { swinging exercises }\end{array}$ & $30 \%$ & 1 & without & 3 \\
\hline
\end{tabular}


Table (8) show time distribution of program content for 6 weeks and as well as distribution of program exercise (appendix 2) , it also shows both exercise intensity and repetition the intra rest time between sets as well as the number of sets.

Post- measurement:

Post- measurement conducted in the period $1 / 3 / 2012$ to $3 / 3 / 2012$

Statistical coefficients and measures used:

- Arithmetic mean
- Standard deviation.

- Median.

- Skewness coefficient

- Correlation coefficient.

- T. Test

- $F$ test.

\section{Results and Discussions}

I - Results of Kinematic Analysis of half roundhouse kick (Mawashi-geri)

Table (9)

Horizontal and vertical distance of body center of gravity Mawashi- geri skill

\begin{tabular}{|c|c|c|c|c|c|}
\hline \multicolumn{6}{|c|}{ Body's center of gravity } \\
\hline Stages & Cadres & Time & $\mathbf{X}$ & $\bar{Y}$ & Pelvis angle \\
\hline \multirow{8}{*}{$\begin{array}{c}1^{\text {st }} \text { stage } \\
\text { (standby position) }\end{array}$} & 1 & $\mathbf{0}$ & 0.6799 & 1.493 & 69 \\
\hline & 2 & 0.04 & 0.684 & 1.493 & 69 \\
\hline & 3 & 0.08 & 0.6955 & 1.491 & 68 \\
\hline & 4 & 0.12 & 0.7081 & 1.489 & 66 \\
\hline & 5 & 0.16 & 0.7321 & 1.483 & 68 \\
\hline & 6 & 0.2 & 0.756 & 1.474 & 73.2 \\
\hline & 7 & 0.24 & 0.7897 & 1.472 & 74.5 \\
\hline & 8 & 0.28 & 0.8242 & 1.469 & 40.8 \\
\hline \multirow{7}{*}{$\begin{array}{c}\mathbf{2}^{\text {nd }} \text { stage } \\
\text { (preliminary stage) }\end{array}$} & 9 & 0.32 & 0.8728 & 1.47 & 36.4 \\
\hline & 10 & 0.36 & 0.8796 & 1.478 & 30.2 \\
\hline & 11 & 0.4 & 0.9051 & 1.49 & 27.8 \\
\hline & 12 & 0.44 & $\mathbf{0 . 9 3 3 4}$ & 1.51 & 27.4 \\
\hline & 13 & 0.48 & 0.9502 & 1.533 & 28.9 \\
\hline & 14 & 0.52 & 0.9633 & 1.547 & 29.3 \\
\hline & 15 & 0.56 & 0.9795 & 1.562 & 64.3 \\
\hline \multirow{4}{*}{$\begin{array}{c}3^{\text {rd }} \text { stage } \\
\text { (first major) }\end{array}$} & 16 & 0.6 & 0.9981 & 1.566 & 67.1 \\
\hline & 17 & 0.64 & 1.014 & 1.572 & 86.3 \\
\hline & 18 & 0.68 & 1.026 & 1.578 & 92.5 \\
\hline & 19 & 0.72 & 1.032 & 1.586 & 119.4 \\
\hline \multirow{9}{*}{$\begin{array}{c}4^{\text {th }} \text { stage } \\
\text { (second major) }\end{array}$} & 20 & 0.76 & 1.043 & 1.54 & 99.4 \\
\hline & 21 & 0.8 & 1.049 & 1.526 & 96.4 \\
\hline & 22 & 0.84 & 1.052 & 1.52 & 88.3 \\
\hline & 23 & 0.88 & 1.062 & 1.497 & 84.6 \\
\hline & 24 & 0.92 & 1.059 & 1.489 & 80 \\
\hline & 25 & 0.96 & 1.061 & 1.492 & 80.2 \\
\hline & 26 & 1 & 1.069 & 1.492 & 80.6 \\
\hline & 27 & 1.04 & 1.063 & 1.501 & 75.4 \\
\hline & 28 & 1.08 & 1.059 & 1.507 & $\begin{array}{l}77.3 \\
\end{array}$ \\
\hline
\end{tabular}




\begin{tabular}{|c|c|c|c|c|c|}
\hline \multicolumn{6}{|c|}{ Body's center of gravity } \\
\hline Stages & Cadres & Time & $\mathbf{X}$ & $\mathbf{Y}$ & Pelvis angle \\
\hline & 29 & 1.12 & 1.056 & 1.519 & 73.8 \\
\hline & 30 & 1.16 & 1.044 & 1.544 & 76 \\
\hline \multirow{20}{*}{$\begin{array}{c}5^{\text {th }} \text { stage } \\
\text { (returning to standby position) }\end{array}$} & 31 & 1.2 & 1.038 & 1.558 & 73 \\
\hline & 32 & 1.24 & 1.025 & 1.572 & 72 \\
\hline & 33 & 1.28 & 1.012 & 1.572 & 50 \\
\hline & 34 & 1.32 & 1.004 & 1.557 & 30 \\
\hline & 35 & 1.36 & 0.9977 & 1.538 & 22.3 \\
\hline & 36 & 1.4 & 0.9868 & 1.511 & 21.8 \\
\hline & 37 & 1.44 & 0.9676 & 1.495 & 29 \\
\hline & 38 & 1.48 & 0.9426 & 1.48 & 28.3 \\
\hline & 39 & 1.52 & 0.9234 & 1.468 & 29.1 \\
\hline & 40 & 1.56 & 0.8981 & 1.466 & 28 \\
\hline & 41 & 1.6 & 0.8662 & 1.472 & 26.8 \\
\hline & 42 & 1.64 & $\mathbf{0 . 8 3 8 1}$ & 1.475 & 36.7 \\
\hline & 43 & 1.68 & 0.8091 & 1.471 & 41.6 \\
\hline & 44 & 1.72 & 0.7746 & 1.471 & 50.1 \\
\hline & 45 & 1.76 & 0.749 & 1.473 & 53.3 \\
\hline & 46 & 1.8 & 0.7314 & 1.475 & 54.3 \\
\hline & 47 & 1.84 & 0.7132 & 1.475 & 52.8 \\
\hline & 48 & 1.88 & 0.698 & 1.478 & 54.8 \\
\hline & 49 & 1.92 & 0.6867 & 1.481 & 56.4 \\
\hline & 50 & 1.96 & 0.6799 & 1.485 & 57.4 \\
\hline
\end{tabular}

- Table (9) lowest body center of gravity Mawashi-geri performance on vertical axis were in two points:

The first was (1.469) at the end of the first stage (standby position). Which shows that player take a suitable position for which leads to decrease the vertical distance of body's center of gravity, giving it stability of balance and exploit fixed leg muscles power in the rush forward and overcome the forces of gravity. (Figure. 1)

- The second was (1.466) in the middle of the fifth stage (returning to standby position. In order to preserve body balance again after beating stage and this is consistent with terms of considering the kick (return back to original position after beating while maintaining body balance (Zanshin). (Figure 2) 


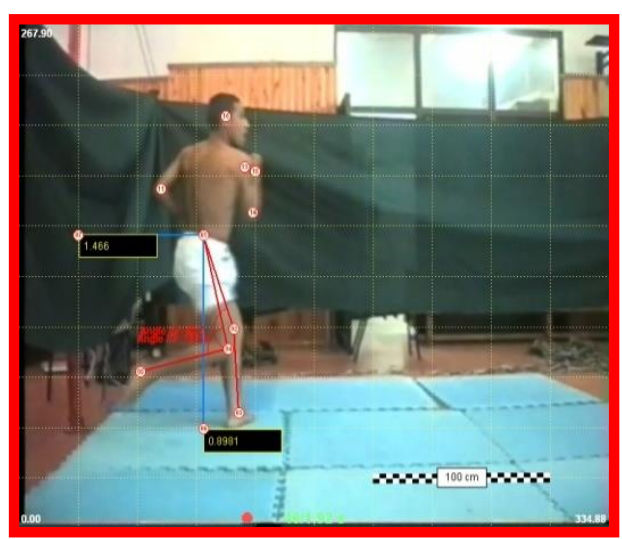

Figure (1)

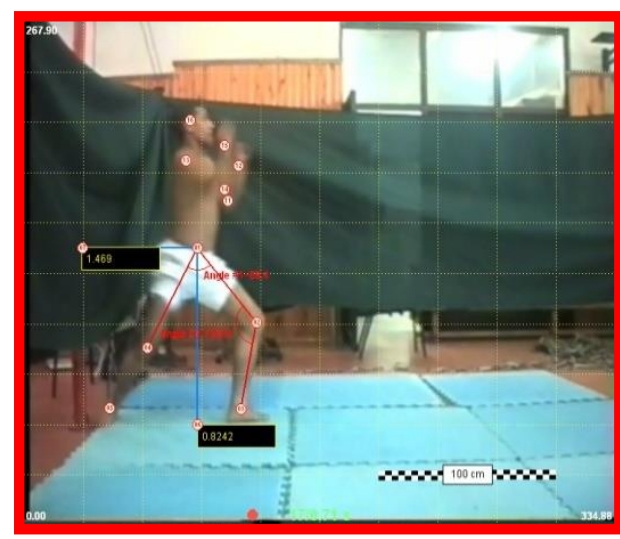

Figure (2)

- As we find the highest body's center of the end of the third stage (Beating stage) to gravity (1.586) during the performance of a arrive to target and high raise of center of free-kick on the vertical axis circular was at gravity of strike foot. (Fig. 3)

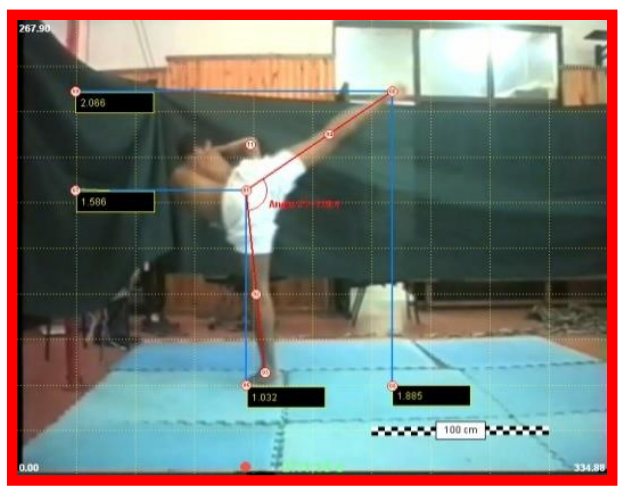

Figure (3)

- Also it is noted the biggest move of body's this stage in matches. That is due to player is center of gravity one side and transverse axis forced to exert another muscle effort to return (1.069) in the fourth stage (major Phase II). with strike leg to back and overcome the Stage for player starting to return standby gravity forces and the force resulting from position. This explains why most of players the body inertia. (Figure 4) resort to go down front with strike leg during

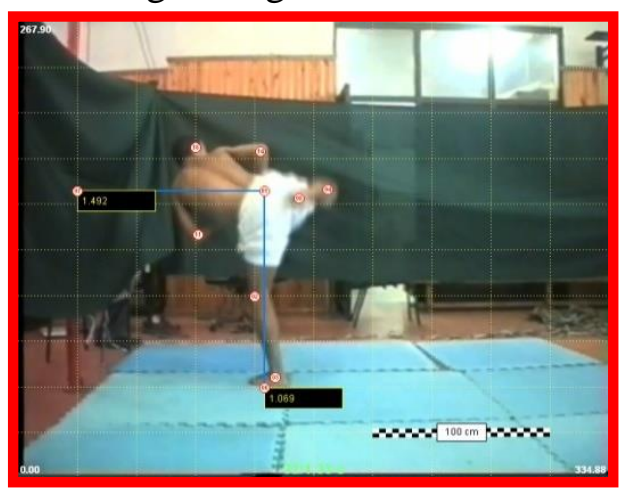

Figure (4) 
- (Table 9) reveals that the highest value of pelvis angle through performance stages of was (119.4 degrees) during the moment of beatings at the end of the third phase (Fig. 4). It's also was at lowest value during second stage (27.4) (Figure 17). And fifth stage

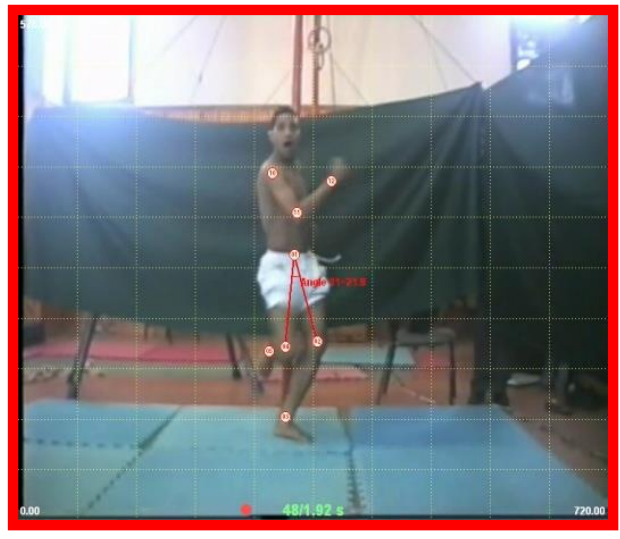

Figure (5)
(21.8 degrees) (Figure 5), where knees are very close to each other during these two phases. This is what distinguishes roundhouse kick for karate players from any other combat sport.

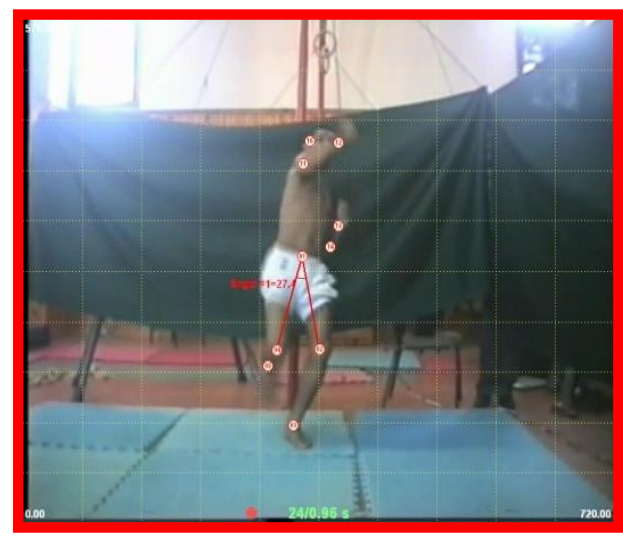

Figure (6)

Table (10)

Horizontal and vertical distance of Strike leg's center of gravity Mawashi- geri skill

\begin{tabular}{|c|c|c|c|c|c|c|}
\hline \multirow{2}{*}{ Stages } & \multirow{2}{*}{ Cadres } & \multirow{2}{*}{ Time } & \multicolumn{2}{|c|}{$\begin{array}{c}\text { Strike leg's center of } \\
\text { gravity }\end{array}$} & \multirow{2}{*}{$\begin{array}{l}\text { Fixed } \\
\text { Knee } \\
\text { angle }\end{array}$} & \multirow{2}{*}{$\begin{array}{c}\text { Strike } \\
\text { knee } \\
\text { Angle }\end{array}$} \\
\hline & & & $\mathbf{X}$ & $Y$ & & \\
\hline \multirow{8}{*}{$\begin{array}{c}1^{\text {st }} \text { stage } \\
\text { (standby position) }\end{array}$} & 1 & $\mathbf{0}$ & 0.383 & 0.043 & 143.6 & 138.6 \\
\hline & 2 & 0.04 & 0.3891 & 0.043 & 142.5 & 138.8 \\
\hline & 3 & 0.08 & 0.3846 & 0.044 & $\begin{array}{l}143.2 \\
\end{array}$ & 138 \\
\hline & 4 & 0.12 & 0.3871 & 0.056 & 145.3 & 138 \\
\hline & 5 & 0.16 & 0.3781 & 0.071 & 148.7 & 140 \\
\hline & 6 & 0.2 & 0.3708 & 0.099 & 143.5 & 137 \\
\hline & 7 & 0.24 & 0.3757 & 0.23 & 132.5 & 136 \\
\hline & 8 & 0.28 & 0.3683 & 0.4 & 130.3 & 140 \\
\hline \multirow{7}{*}{$\begin{array}{c}2^{\text {nd }} \text { stage } \\
\text { (preliminary stage) }\end{array}$} & 9 & 0.32 & 0.3955 & 0.5625 & 128.9 & 140 \\
\hline & 10 & 0.36 & 0.4807 & 0.614 & 129.3 & 130 \\
\hline & 11 & 0.4 & 0.6489 & 0.6577 & 130.2 & 123.9 \\
\hline & 12 & 0.44 & 0.8786 & 0.7177 & 136.5 & 103.7 \\
\hline & 13 & 0.48 & 1.153 & 0.8596 & 148.4 & 91.3 \\
\hline & 14 & 0.52 & 1.274 & 0.9776 & 153.1 & 79.1 \\
\hline & 15 & 0.56 & 1.467 & 1.255 & 153.4 & 49.1 \\
\hline \multirow{4}{*}{$\begin{array}{c}3^{\text {rd }} \text { stage } \\
\text { (first major) }\end{array}$} & 16 & 0.6 & 1.708 & 1.684 & 161.3 & $\begin{array}{l}55.2 \\
\end{array}$ \\
\hline & 17 & 0.64 & 1.815 & 1.877 & 170.1 & 80.4 \\
\hline & 18 & 0.68 & 1.884 & 1.989 & 175 & 128.2 \\
\hline & 19 & 0.72 & 1.885 & 2.066 & 177.1 & 179 \\
\hline \multirow{3}{*}{$\begin{array}{c}4^{\text {th }} \text { stage } \\
\text { (second major ) }\end{array}$} & 20 & 0.76 & 1.85 & 2.06 & 176.6 & 115 \\
\hline & 21 & 0.8 & 1.739 & 1.956 & 176.4 & 79.2 \\
\hline & 22 & 0.84 & 1.591 & 1.823 & 175.2 & 45.8 \\
\hline
\end{tabular}




\begin{tabular}{|c|c|c|c|c|c|c|}
\hline \multirow[t]{2}{*}{ Stages } & \multirow[t]{2}{*}{ Cadres } & \multirow[t]{2}{*}{ Time } & \multicolumn{2}{|c|}{$\begin{array}{c}\text { Strike leg's center of } \\
\text { gravity }\end{array}$} & \multirow{2}{*}{$\begin{array}{l}\text { Fixed } \\
\text { Knee } \\
\text { angle }\end{array}$} & \multirow{2}{*}{$\begin{array}{c}\text { Strike } \\
\text { knee } \\
\text { Angle }\end{array}$} \\
\hline & & & $\mathbf{X}$ & $\mathbf{Y}$ & & \\
\hline & 23 & 0.88 & 1.54 & 1.576 & 175 & 30.8 \\
\hline & 24 & 0.92 & 1.505 & 1.532 & 172 & \\
\hline & 25 & 0.96 & 1.562 & 1.598 & 167.1 & 41.1 \\
\hline & 26 & 1 & 1.678 & 1.588 & 172.2 & 60.1 \\
\hline & 27 & 1.04 & 1.749 & 1.521 & 168.7 & 75.4 \\
\hline & 28 & 1.08 & 1.753 & 1.42 & 165 & 82.6 \\
\hline & 29 & 1.12 & 1.677 & 1.314 & 164 & 80.2 \\
\hline & 30 & 1.16 & 1.568 & 1.193 & 154 & 63 \\
\hline \multirow{20}{*}{$\begin{array}{c}5^{\text {th }} \text { stage } \\
\text { (returning to standby position) }\end{array}$} & 31 & 1.2 & 1.421 & 1.121 & 162.9 & 42.2 \\
\hline & 32 & 1.24 & 1.297 & 1.089 & 166.2 & 40 \\
\hline & 33 & 1.28 & 1.193 & 1.03 & 168.6 & 60.4 \\
\hline & 34 & 1.32 & 1.089 & 0.9607 & 165.6 & 70.8 \\
\hline & 35 & 1.36 & 0.9989 & 0.9011 & 165.2 & 96.7 \\
\hline & 36 & 1.4 & 0.8903 & 0.8302 & 164.5 & 115.5 \\
\hline & 37 & 1.44 & $\mathbf{0 . 7 8 3 7}$ & 0.7622 & 160.4 & 153.2 \\
\hline & 38 & 1.48 & 0.6494 & 0.7137 & 139.8 & 85.9 \\
\hline & 39 & 1.52 & 0.5267 & 0.6705 & 146.9 & 72.7 \\
\hline & 40 & 1.56 & 0.4348 & 0.6007 & 146.4 & 68.4 \\
\hline & 41 & 1.6 & 0.4035 & 0.5465 & 148.6 & 66.2 \\
\hline & 42 & 1.64 & 0.3596 & 0.5199 & 145.7 & 81.1 \\
\hline & 43 & 1.68 & 0.3526 & 0.5179 & 153.4 & 70.7 \\
\hline & 44 & 1.72 & 0.3477 & 0.5154 & 154.5 & 119.2 \\
\hline & 45 & 1.76 & 0.3403 & 0.5081 & 152.7 & 133 \\
\hline & 46 & 1.8 & 0.3428 & 0.4982 & 151.2 & 134 \\
\hline & 47 & 1.84 & 0.3428 & 0.4982 & 153.1 & 134.5 \\
\hline & 48 & 1.88 & 0.3453 & 0.4884 & 155.3 & 136.5 \\
\hline & 49 & 1.92 & 0.3453 & 0.4884 & 158.8 & 136.8 \\
\hline & 50 & 1.96 & 0.3502 & 0.481 & 160.2 & 138 \\
\hline
\end{tabular}

(Table 10) shows that:

Highest vertical distance of strike feet's center of gravity (2.066) and also the longest horizontal distance (1.885) at the end of the third stage (Beating moment). Which indicate large motor range for strike leg's foot and kick's height. (Figure 7)

- Knee angle measurements for fixed leg during performance ranged between (128.9 degrees) and (177.1 degrees). (Figure 19) (Figure 8).

- Knee angle measurements for strike leg Had the highest measurement during beating moment where the amounted to (179.5 degrees), where the lowest measurement (26.4 degrees) was during the fourth stage. Thus have a physics importance as foot approaching body basing axis greatly facilitate body return process and turning to return to original position. It is also has technical importance where it is one of kick considering condition quick bending of strike leg after the beating. (Figure 9) (Figure 10) 


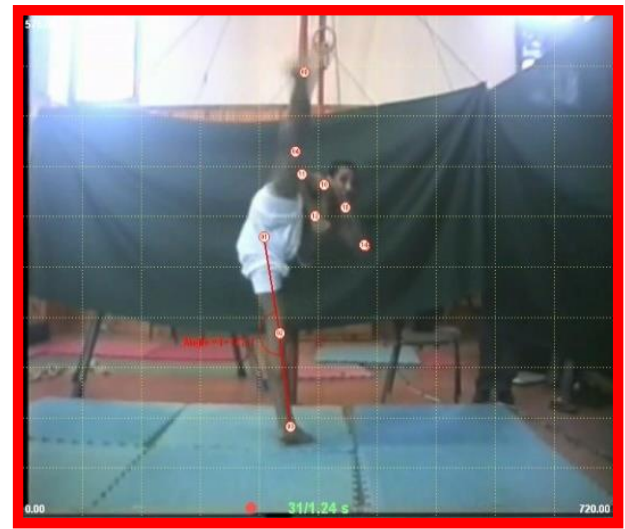

Figure (7)

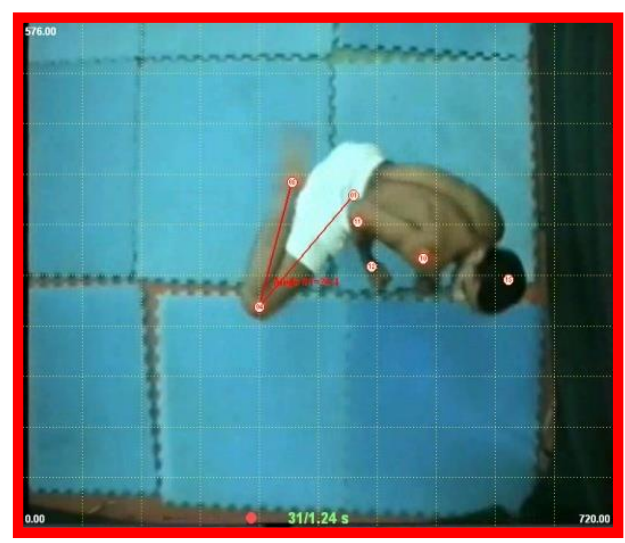

Figure (9)

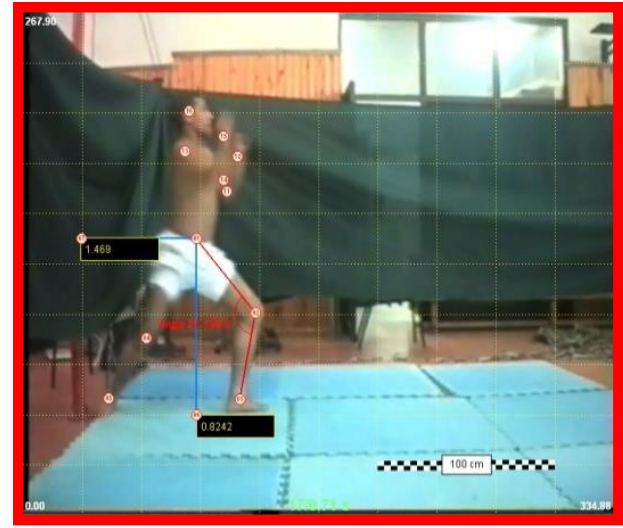

Figure (8)

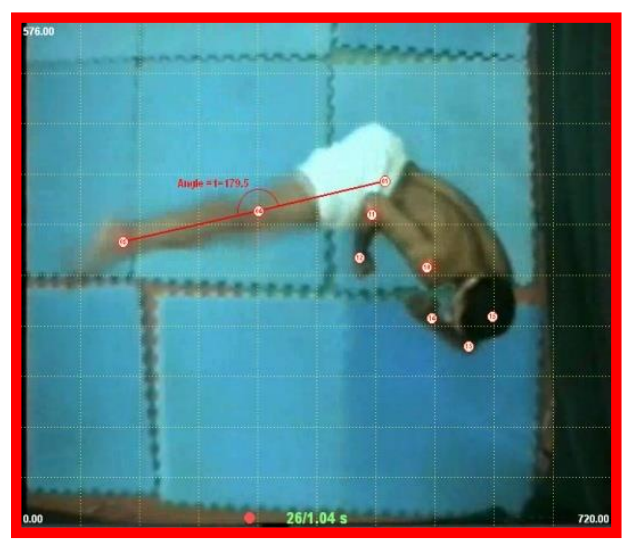

Figure (10)

Table (11)

Velocity resultant of (Body - Strike leg - Strike leg foot) Center of Gravity

\begin{tabular}{|c|c|c|c|c|c|}
\hline \multirow{3}{*}{ Stages } & \multirow{3}{*}{ Cadres } & \multirow{3}{*}{ Time } & $\begin{array}{c}\text { Body Center } \\
\text { of Gravity }\end{array}$ & $\begin{array}{c}\text { Strike leg's } \\
\text { Center of Gravity }\end{array}$ & $\begin{array}{l}\text { Strike leg's foot } \\
\text { Center of Gravity }\end{array}$ \\
\hline & & & v_mag & v_mag & v_mag \\
\hline & & & {$[\mathrm{m} / \mathrm{s}]$} & {$[\mathrm{m} / \mathrm{s}]$} & {$[\mathrm{m} / \mathrm{s}]$} \\
\hline \multirow{8}{*}{$\begin{array}{c}1^{\text {st }} \text { stage } \\
\text { (standby position) }\end{array}$} & 1 & 0 & 0.0883 & 0.2204 & 0.09425 \\
\hline & 2 & 0.04 & 0.1975 & 0.1808 & 0.02737 \\
\hline & 3 & 0.08 & 0.3082 & 0.1455 & 0.03964 \\
\hline & 4 & 0.12 & 0.4687 & 0.328 & 0.08081 \\
\hline & 5 & 0.16 & 0.6238 & 0.6103 & 0.2037 \\
\hline & 6 & 0.2 & 0.7326 & 0.8031 & 0.03073 \\
\hline & 7 & 0.24 & 0.8557 & 1.22 & 0.04765 \\
\hline & 8 & 0.28 & 1.04 & 1.82 & 0.9163 \\
\hline \multirow{5}{*}{$\begin{array}{c}2^{\text {nd }} \text { stage } \\
\text { (preliminary stage) }\end{array}$} & 9 & 0.32 & 0.7004 & 2.41 & 2.048 \\
\hline & 10 & 0.36 & 0.4696 & 2.781 & 3.384 \\
\hline & 11 & 0.4 & 0.7832 & 3.492 & 5.14 \\
\hline & 12 & 0.44 & 0.7795 & 4.106 & 6.787 \\
\hline & 13 & 0.48 & 0.5993 & 3.585 & 5.917 \\
\hline
\end{tabular}




\begin{tabular}{|c|c|c|c|c|c|}
\hline \multirow{3}{*}{ Stages } & \multirow{3}{*}{ Cadres } & \multirow{3}{*}{ Time } & $\begin{array}{c}\text { Body Center } \\
\text { of Gravity }\end{array}$ & $\begin{array}{c}\text { Strike leg's } \\
\text { Center of Gravity }\end{array}$ & $\begin{array}{l}\text { Strike leg's foot } \\
\text { Center of Gravity }\end{array}$ \\
\hline & & & v_mag & v_mag & v_mag \\
\hline & & & {$[\mathrm{m} / \mathrm{s}]$} & {$[\mathrm{m} / \mathrm{s}]$} & {$[\mathrm{m} / \mathrm{s}]$} \\
\hline & 14 & 0.52 & 0.5225 & 3.394 & 6.317 \\
\hline & 15 & 0.56 & 0.4959 & 3.458 & 8.88 \\
\hline \multirow{4}{*}{$\begin{array}{c}3^{\text {rd }} \text { stage } \\
\text { (first major) }\end{array}$} & 16 & 0.6 & 0.4473 & 2.952 & 10.36 \\
\hline & 17 & 0.64 & 0.3454 & 2.122 & 8.9 \\
\hline & 18 & 0.68 & 0.2993 & 0.8555 & 2.352 \\
\hline & 19 & 0.72 & 0.4176 & 0.1544 & 0.9791 \\
\hline \multirow{11}{*}{$\begin{array}{c}4^{\text {th }} \text { stage } \\
\text { (second major) }\end{array}$} & 20 & 0.76 & 0.4343 & 0.9227 & 2.066 \\
\hline & 21 & 0.8 & 0.2677 & 1.261 & 4.39 \\
\hline & 22 & 0.84 & 0.3865 & 1.887 & 5.366 \\
\hline & 23 & 0.88 & 0.4086 & 1.892 & 3.793 \\
\hline & 24 & 0.92 & 0.0744 & 0.6024 & 0.3839 \\
\hline & 25 & 0.96 & 0.1268 & 0.5065 & 2.277 \\
\hline & 26 & 1 & 0.1189 & 0.255 & 2.532 \\
\hline & 27 & 1.04 & 0.2234 & 0.4209 & 2.296 \\
\hline & 28 & 1.08 & 0.2491 & 0.8321 & 2.74 \\
\hline & 29 & 1.12 & 0.5021 & 0.9261 & 3.662 \\
\hline & 30 & 1.16 & 0.5346 & 1.091 & 4.009 \\
\hline \multirow{20}{*}{$\begin{array}{c}5^{\text {th }} \text { stage } \\
\text { (returning to standby position) }\end{array}$} & 31 & 1.2 & 0.422 & 1.136 & 3.633 \\
\hline & 32 & 1.24 & 0.3668 & 1.189 & 3.067 \\
\hline & 33 & 1.28 & 0.3274 & 1.39 & 3.049 \\
\hline & 34 & 1.32 & 0.4693 & 1.47 & 2.909 \\
\hline & 35 & 1.36 & 0.6043 & 1.912 & 2.972 \\
\hline & 36 & 1.4 & 0.65 & 2.173 & 3.202 \\
\hline & 37 & 1.44 & 0.6742 & 2.077 & 3.345 \\
\hline & 38 & 1.48 & 0.6513 & 1.93 & 3.41 \\
\hline & 39 & 1.52 & 0.585 & 1.983 & 3.032 \\
\hline & 40 & 1.56 & 0.7171 & 2.059 & 2.185 \\
\hline & 41 & 1.6 & 0.7585 & 1.78 & 1.379 \\
\hline & 42 & 1.64 & 0.7145 & 1.463 & 0.7297 \\
\hline & 43 & 1.68 & 0.7956 & 1.455 & 0.1581 \\
\hline & 44 & 1.72 & 0.7512 & 1.236 & 0.1968 \\
\hline & 45 & 1.76 & 0.5411 & 0.7825 & 0.2237 \\
\hline & 46 & 1.8 & 0.4476 & 0.5771 & 0.1267 \\
\hline & 47 & 1.84 & 0.4206 & 0.4203 & 0.1267 \\
\hline & 48 & 1.88 & 0.3413 & 0.2619 & 0.1267 \\
\hline & 49 & 1.92 & 0.2422 & 0.1254 & 0.1108 \\
\hline & 50 & 1.96 & 0.151 & 0.0462 & 0.2477 \\
\hline
\end{tabular}




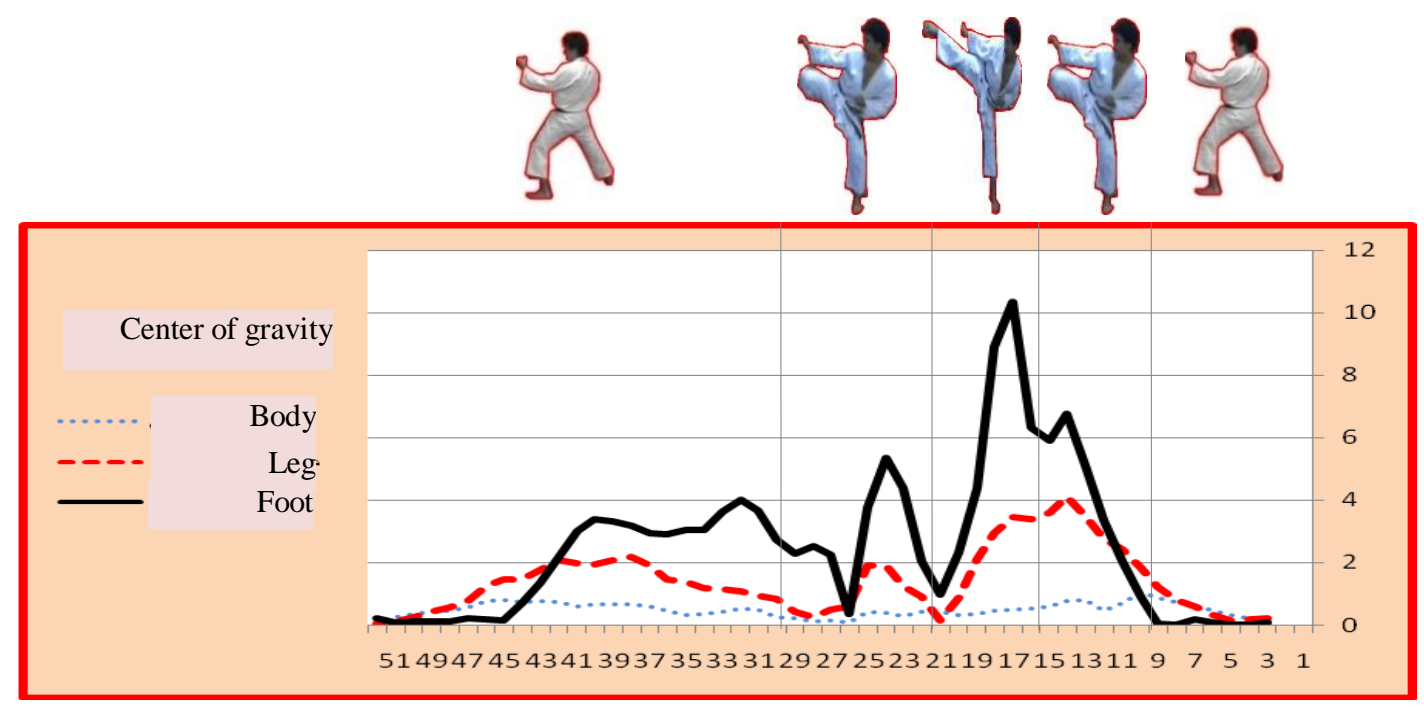

Figure (11)

Speed resultant curve of (Body - Strike leg - Strike leg foot) Center of Gravity

From speed velocity figure (11) it is possible to note the following

- Through the velocity curve shape (11): note the following:

1- Highest velocity during performance was in favor foot center of gravity as it represents end of the Kinematic open string here, and thus velocity of ankle and knee joints is the largest due to large motor range for them.

2- Velocity is too large in second (knee bent) and third phases (movement stopping) and faster than velocity in fourth and fifth phases, for all strike leg joints i.e. foot faster during reaching target.
3-At beating moment foot velocity almost approaching but not equal to zero (movement stopping) and it shows the big foot return velocity. This is consistent with technical characteristics for kumite players (technique) where within performance terms that foot touches face only without any injury. Unlike the velocity of leg's center of gravity which is very close to zero at this moment; to maintain foot instep height at this level.

4-Foot velocity significantly decrease at returning to original position velocity due to players' focus on large knee largest to reach first fight position and keeping body's balance. 
Table (12)

Acceleration resultant of (Body - Strike leg - Strike leg foot) Center of Gravity

\begin{tabular}{|c|c|c|c|c|c|}
\hline \multirow{3}{*}{ Stages } & \multirow{3}{*}{ Cadres } & \multirow{3}{*}{ Time } & $\begin{array}{c}\text { Body Center } \\
\text { of Gravity }\end{array}$ & $\begin{array}{c}\text { Strike leg's } \\
\text { Center of Gravity }\end{array}$ & $\begin{array}{l}\text { Strike leg's foot } \\
\text { Center of Gravity }\end{array}$ \\
\hline & & & a_mag & a_mag & a_mag \\
\hline & & & {$\left[\mathrm{m} / \mathrm{s}^{\wedge} 2\right]$} & {$\left[\mathrm{m} / \mathrm{s}^{\wedge} 2\right]$} & {$\left[\mathrm{m} / \mathrm{s}^{\wedge} 2\right]$} \\
\hline \multirow{8}{*}{$\begin{array}{c}1^{\text {st }} \text { stage } \\
\text { (standby position) }\end{array}$} & 1 & 0 & & & \\
\hline & 2 & 0.04 & & & \\
\hline & 3 & 0.08 & 3.378 & 2.38 & 1.668 \\
\hline & 4 & 0.12 & 3.421 & 4.145 & 0.5654 \\
\hline & 5 & 0.16 & 3.425 & 6.628 & 0.05918 \\
\hline & 6 & 0.2 & 3.64 & 9.089 & 5.278 \\
\hline & 7 & 0.24 & 2.022 & 11.88 & 13.25 \\
\hline & 8 & 0.28 & 3.055 & 14.26 & 21.61 \\
\hline \multirow{7}{*}{$\begin{array}{c}2^{\text {nd }} \text { stage } \\
\text { (preliminary stage) }\end{array}$} & 9 & 0.32 & 3.561 & 16.97 & 32.79 \\
\hline & 10 & 0.36 & 4.364 & 19.09 & 39.65 \\
\hline & 11 & 0.4 & 2.868 & 15.75 & 27.02 \\
\hline & 12 & 0.44 & 1.08 & 16.52 & 23.95 \\
\hline & 13 & 0.48 & 1.985 & 16.66 & 44.95 \\
\hline & 14 & 0.52 & 2.73 & 14.01 & 38.86 \\
\hline & 15 & 0.56 & 2.831 & 11.18 & 16.16 \\
\hline \multirow{4}{*}{$\begin{array}{c}3^{\text {rd }} \text { stage } \\
\text { (first major) }\end{array}$} & 16 & 0.6 & 3.58 & 16.18 & 32.29 \\
\hline & 17 & 0.64 & 4.018 & 23.05 & 64.35 \\
\hline & 18 & 0.68 & 3.59 & 24.68 & 65.19 \\
\hline & 19 & 0.72 & 2.223 & 21.17 & 54.04 \\
\hline \multirow{11}{*}{$\begin{array}{c}4^{\text {th }} \text { stage } \\
\text { (second major) }\end{array}$} & 20 & 0.76 & 0.8671 & 16.61 & 49.43 \\
\hline & 21 & 0.8 & 0.8259 & 11.03 & 30.69 \\
\hline & 22 & 0.84 & 1.875 & 3.163 & 15.66 \\
\hline & 23 & 0.88 & 2.036 & 8.723 & 43.98 \\
\hline & 24 & 0.92 & 3.328 & 11.58 & 41.94 \\
\hline & 25 & 0.96 & 3.669 & 9.655 & 15.69 \\
\hline & 26 & 1 & 2.071 & 5.184 & 21.77 \\
\hline & 27 & 1.04 & 3.117 & 7.526 & 36.82 \\
\hline & 28 & 1.08 & 2.876 & 6.709 & 36.57 \\
\hline & 29 & 1.12 & 1.547 & 4.578 & 27.66 \\
\hline & 30 & 1.16 & 1.418 & 2.698 & 16.1 \\
\hline \multirow{11}{*}{$\begin{array}{c}5^{\text {th }} \text { stage } \\
\text { (returning to standby position) }\end{array}$} & 31 & 1.2 & 4.123 & 3.405 & 8.864 \\
\hline & 32 & 1.24 & 5.879 & 4.431 & 6.753 \\
\hline & 33 & 1.28 & 5.974 & 6.312 & 6.178 \\
\hline & 34 & 1.32 & 4.461 & 6.677 & 3.437 \\
\hline & 35 & 1.36 & 2.244 & 5.191 & 2.756 \\
\hline & 36 & 1.4 & 2.687 & 5.752 & 5.839 \\
\hline & 37 & 1.44 & 3.191 & 7.386 & 2.879 \\
\hline & 38 & 1.48 & 3.951 & 8.832 & 6.877 \\
\hline & 39 & 1.52 & 3.615 & 7.509 & 14.09 \\
\hline & 40 & 1.56 & 2.603 & 5.287 & 17.55 \\
\hline & 41 & 1.6 & 1.441 & 5.313 & 17.87 \\
\hline
\end{tabular}




\begin{tabular}{|c|c|c|c|c|c|}
\hline \multirow{3}{*}{ Stages } & \multirow{3}{*}{ Cadres } & \multirow{3}{*}{ Time } & $\begin{array}{c}\text { Body Center } \\
\text { of Gravity }\end{array}$ & $\begin{array}{c}\text { Strike leg's } \\
\text { Center of Gravity }\end{array}$ & $\begin{array}{c}\text { Strike leg's foot } \\
\text { Center of Gravity }\end{array}$ \\
\hline & & & a_mag & a_mag & a_mag \\
\hline & & & {$\left[\mathrm{m} / \mathrm{s}^{\wedge} \mathbf{2}\right]$} & {$\left[\mathrm{m} / \mathrm{s}^{\wedge} \mathbf{2}\right]$} & {$\left[\mathrm{m} / \mathrm{s}^{\wedge} \mathbf{2}\right]$} \\
\hline & 42 & 1.64 & 0.5033 & 5.472 & 12.83 \\
\hline & 43 & 1.68 & 1.13 & 5.831 & 7.282 \\
\hline & 44 & 1.72 & 1.901 & 5.95 & 3.902 \\
\hline & 45 & 1.76 & 2.59 & 6.706 & 1.323 \\
\hline & 46 & 1.8 & 2.495 & 5.888 & 1.162 \\
\hline & 47 & 1.84 & 1.915 & 4.135 & 0.9452 \\
\hline & 48 & 1.88 & 1.96 & 3.448 & 0.7124 \\
\hline & 49 & 1.92 & 2.117 & 2.954 & 0.5066 \\
\hline & 50 & 1.96 & 1.951 & 1.89 & 0.6204 \\
\hline
\end{tabular}

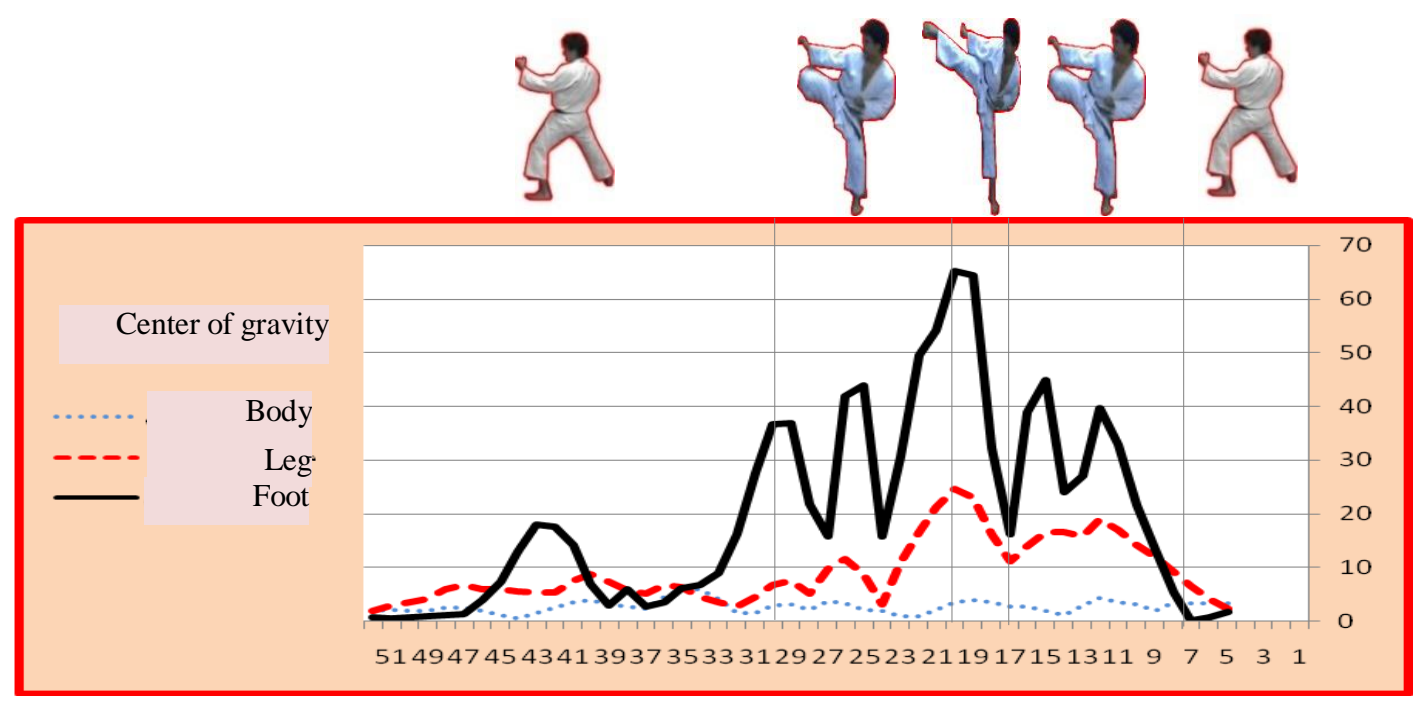

Figure (12)

\section{Acceleration resultant of (Body - Strike} leg - Strike leg foot) Center of Gravity

- From acceleration curve (Figure 12) it is possible to note the following:

1- Highest acceleration value in foot movement was during third stage $\left(1^{\text {st }}\right.$ main stage ), which precedes foot accessing target, which shows the optimal use of muscle forces in the best way to give the maximum incremental speed, where it to reach maximum acceleration speed all forces should effect in sequences in the expected time directly in the same movement line.
2- The Highest acceleration of body's center of gravity movement was in fifth stage (return to original position) shows body's center plays leadership role of player's return movement, where coaches should be aware of this role to body rotation and change ding trends from one stage to another during the motor performance Kick skill circula 
Table (13)

Force resultant of (Body - Strike leg - Strike leg foot) Center of Gravity

\begin{tabular}{|c|c|c|c|c|c|}
\hline \multirow{3}{*}{ Stages } & \multirow{3}{*}{ Cadres } & \multirow{3}{*}{ Time } & $\begin{array}{c}\text { Body Center } \\
\text { of Gravity }\end{array}$ & $\begin{array}{c}\text { Strike leg's } \\
\text { Center of Gravity }\end{array}$ & $\begin{array}{l}\text { Strike leg's foot } \\
\text { Center of Gravity }\end{array}$ \\
\hline & & & F_mag & F_mag & F_mag \\
\hline & & & {$[\mathrm{N}]$} & {$[\mathbf{N}]$} & {$[\mathbf{N}]$} \\
\hline \multirow{8}{*}{$\begin{array}{c}1^{\text {st }} \text { stage } \\
\text { (standby position) }\end{array}$} & 1 & $\mathbf{0}$ & & & \\
\hline & 2 & 0.04 & & & \\
\hline & 3 & $\begin{array}{l}0.08 \\
\end{array}$ & 202.7 & 22.99 & 1.501 \\
\hline & 4 & 0.12 & 205.2 & 40.04 & 0.5088 \\
\hline & 5 & 0.16 & 205.5 & 64.03 & $\mathbf{0 . 0 5 3 2 6}$ \\
\hline & 6 & 0.2 & 218.4 & 87.8 & 4.75 \\
\hline & 7 & 0.24 & 121.3 & 114.8 & 11.93 \\
\hline & 8 & 0.28 & \begin{tabular}{ll|}
183.3 \\
\end{tabular} & 137.7 & 19.45 \\
\hline \multirow{7}{*}{$\begin{array}{c}2^{\text {nd }} \text { stage } \\
\text { (preliminary stage) }\end{array}$} & 9 & 0.32 & 213.6 & 163.9 & 29.51 \\
\hline & 10 & 0.36 & 261.9 & 184.4 & 35.69 \\
\hline & 11 & 0.4 & 172.1 & 152.2 & 24.32 \\
\hline & 12 & 0.44 & 64.82 & 159.6 & 21.55 \\
\hline & 13 & $\begin{array}{l}0.48 \\
\end{array}$ & 119.1 & 160.9 & 40.45 \\
\hline & 14 & 0.52 & 163.8 & 135.4 & 34.97 \\
\hline & 15 & 0.56 & 169.9 & 108 & 14.55 \\
\hline \multirow{4}{*}{$\begin{array}{c}3^{\text {rd }} \text { stage } \\
\text { (first major) }\end{array}$} & 16 & 0.6 & 214.8 & 156.3 & 29.06 \\
\hline & 17 & 0.64 & 241.1 & 222.6 & 57.91 \\
\hline & 18 & 0.68 & 215.4 & 238.4 & 58.67 \\
\hline & 19 & 0.72 & 133.4 & 204.5 & 48.64 \\
\hline \multirow{11}{*}{$\begin{array}{c}4^{\text {th }} \text { stage } \\
\text { (second major) }\end{array}$} & 20 & 0.76 & 52.02 & 160.4 & 44.49 \\
\hline & 21 & 0.8 & 49.56 & 106.5 & 27.62 \\
\hline & 22 & 0.84 & 112.5 & $\begin{array}{l}30.55 \\
\end{array}$ & 14.1 \\
\hline & 23 & 0.88 & 122.1 & 84.27 & $\begin{array}{l}39.58 \\
\end{array}$ \\
\hline & 24 & 0.92 & 199.7 & 111.8 & 37.75 \\
\hline & 25 & 0.96 & 220.1 & 93.27 & $\begin{array}{l}14.13 \\
\end{array}$ \\
\hline & 26 & 1 & 124.3 & $\begin{array}{l}50.08 \\
\end{array}$ & 19.59 \\
\hline & 27 & 1.04 & 187 & 72.71 & 33.14 \\
\hline & 28 & 1.08 & 172.5 & $\begin{array}{l}64.81 \\
\end{array}$ & 32.91 \\
\hline & 29 & 1.12 & $\begin{array}{l}92.8 \\
\end{array}$ & 444.23 & 24.9 \\
\hline & 30 & 1.16 & 85.06 & 26.06 & 14.49 \\
\hline \multirow{17}{*}{$\begin{array}{c}5^{\text {th }} \text { stage } \\
\text { (returning to standby position) }\end{array}$} & 31 & 1.2 & 247.4 & 32.89 & $\begin{array}{l}7.978 \\
\end{array}$ \\
\hline & 32 & 1.24 & $\begin{array}{l}352.7 \\
\end{array}$ & 42.8 & $\begin{array}{l}6.078 \\
\end{array}$ \\
\hline & 33 & 1.28 & $\begin{array}{l}358.4 \\
\end{array}$ & 60.97 & 5.56 \\
\hline & 34 & 1.32 & 267.7 & 64.5 & 3.094 \\
\hline & 35 & 1.36 & 134.6 & $\begin{array}{l}50.15 \\
\end{array}$ & 2.48 \\
\hline & 36 & 1.4 & 161.2 & $\begin{array}{l}55.56 \\
\end{array}$ & 5.256 \\
\hline & 37 & 1.44 & $\begin{array}{l}191.5 \\
\end{array}$ & 71.35 & 2.591 \\
\hline & 38 & 1.48 & 237.1 & 85.32 & 6.189 \\
\hline & 39 & 1.52 & 216.9 & $\begin{array}{l}72.53 \\
\end{array}$ & 12.68 \\
\hline & 40 & 1.56 & 156.2 & 51.07 & 15.79 \\
\hline & 41 & 1.6 & 86.47 & $\begin{array}{l}51.32 \\
\end{array}$ & 16.09 \\
\hline & 42 & 1.64 & 30.2 & 52.86 & 11.55 \\
\hline & 43 & 1.68 & 67.82 & $\begin{array}{l}56.33 \\
\end{array}$ & 6.554 \\
\hline & 44 & 1.72 & 114.1 & $\begin{array}{l}57.48 \\
\end{array}$ & 3.511 \\
\hline & 45 & 1.76 & 155.4 & $\begin{array}{l}64.78 \\
\end{array}$ & 1.19 \\
\hline & 46 & 1.8 & 149.7 & 56.87 & 1.046 \\
\hline & 47 & 1.84 & 114.9 & 39.95 & 0.8507 \\
\hline
\end{tabular}




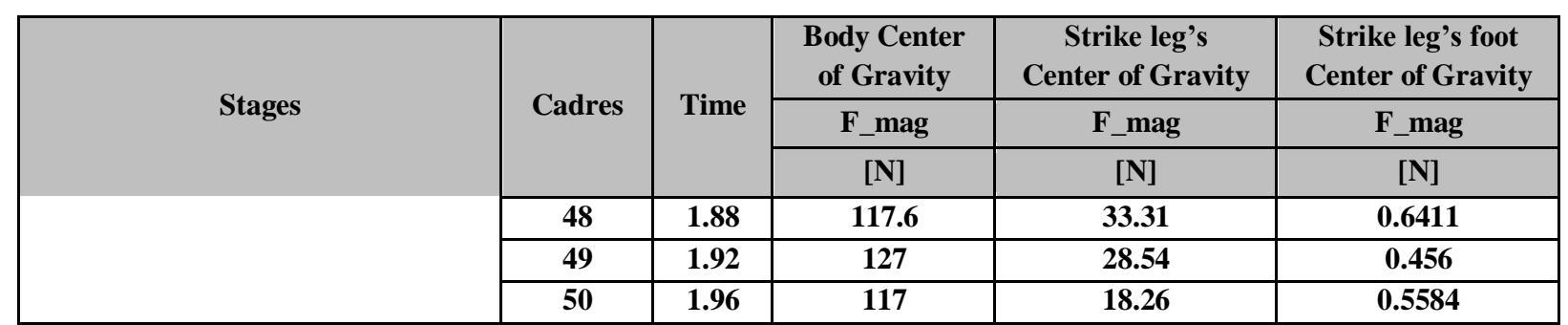

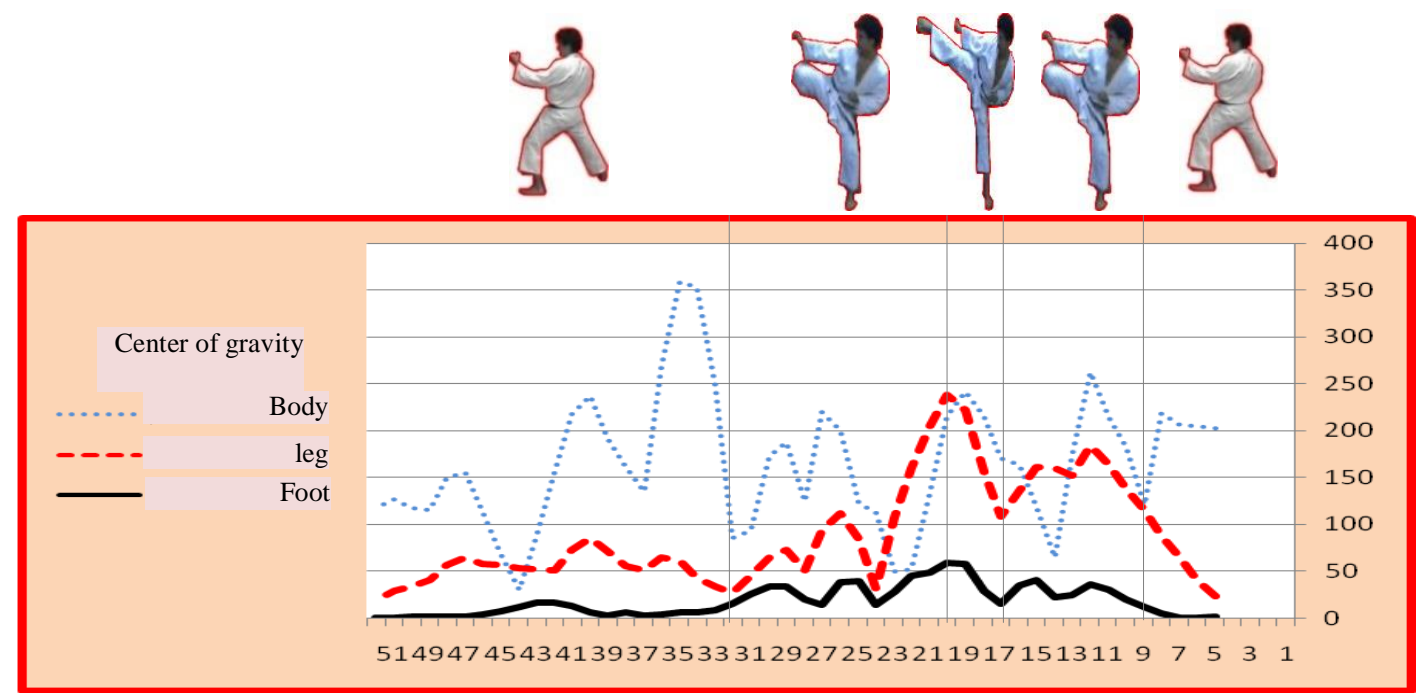

Figure (13)

Force resultant curve of (Body - Strike leg - Strike leg foot) Center of Gravity

- From force curve (figure 13) we note the following:

1- Force increased hesitantly increased from the beginning of till knee raising stage and then getting increased on a regular basis to reach its peak at the end of $1^{\text {st }}$ main Stage when accessing target then reduced hesitantly. Then significantly increased again indicating good distribution of muscle force on leg's various joints.

2- Comparison between the force curves of body and foot centers of gravity that there are significance variance in favor of body's center of gravity, and this explains work muscles involved in this skill performance, as the major force result from the muscles working on the pelvis and thigh joints, as well as back and abdomen muscles.

3- Body's center of gravity bears the brunt hub fulcrum of the forces acting on the body as a whole in the back stage of the original situation. This illustrates the importance of the return stage to (Zanshin) position for kumite players which; which considered by experts one of the basics technique for most basic skills of karate players.

4- Significant rise in force values of Body's center of gravity during the return phase to original position is a result body's resistance to external forces affecting it, which are the generated force of inertia momentum and gravitational forces as a result of the continuous change of body's center of gravity during performance. 
Table (14)

Improvement rate and differences significance between ore and post-measurements in (motor reaction speed - accuracy - fine motor reaction speed) tests

\begin{tabular}{|c|c|c|c|c|c|c|c|c|}
\hline \multirow{2}{*}{ Variables } & \multicolumn{2}{|c|}{$\begin{array}{c}\text { Pre- } \\
\text { measurements }\end{array}$} & \multicolumn{2}{|c|}{$\begin{array}{c}\text { Post- } \\
\text { measurements }\end{array}$} & \multirow{2}{*}{$\begin{array}{c}\text { Mean } \\
\text { differences }\end{array}$} & \multirow{2}{*}{$\begin{array}{c}\text { Mean } \\
\text { Differences }\end{array}$} & \multirow{2}{*}{$\begin{array}{c}\text { SD } \\
\text { Differences }\end{array}$} & \multirow[t]{2}{*}{$\mathbf{T}$} \\
\hline & Mean & SD & Mean & SD & & & & \\
\hline $\begin{array}{c}\text { motor } \\
\text { reaction speed }\end{array}$ & 1.4 & 0.09 & 1.06 & 1.06 & 0.03 & 0.34 & 0.09 & $14.5^{*}$ \\
\hline Accuracy & 1.27 & 1.16 & 3.8 & 1.01 & 1.01 & 2.5 & 0.74 & $13.2 *$ \\
\hline $\begin{array}{l}\text { Fine motor } \\
\text { reaction speed }\end{array}$ & 1.4 & 0.07 & 1.07 & 0.03 & 0.03 & 0.33 & 0.08 & $16.5^{*}$ \\
\hline
\end{tabular}

T significance at $\mathbf{0 . 0 5}$ level $=\mathbf{2} .26$

Table No. (14) results reveal existence of statistically significant differences between pre and post-measurements for experimental group in (motor reaction speed - accuracy value in fine motor reaction speed test; while $\mathrm{T}$ significance at 0.05 level $=2.14$ level and, this shows that there are significant differences in favor of the post measurement of experimental group in variables under consideration.

\section{Conclusions:}

In light of research objectives, its queries, within the limits of research sample and procedures and through results discussion and interpretation it was possible to conclude the following:

- Studying curves ad tables for distances and displacements resulting from kinematic analysis for the skill under study, It is clear that Knee and pelvis joints represent great importance in Mawashi-geri kick for karate players

- Studying curves and tables for velocity, force and acceleration for body, leg, and foot centers of gravity, as well as pelvis and knee angles we conclude that velocity and flexibility are the most important physical fine motor reaction speed) tests as lowest value of $\mathrm{T}$ was (13.2) in accuracy test and (16.5 as

highest

parameters of Mawashi-geri for karate players

- Tables and curves for distances of centers of gravity results illustrated the important and main role played by body's center of gravity in success of performing motor requirement of Mawashi-geri for karate players.

- Exercises similar to skill developed considering kinetic analysis results according to its impact force resulting from the linear relationship between them and mechanical determinants resulted from kinetic analysis.

- Results of this research showed that using the proposed training program improved motor reaction speed and accuracy through a relative improvement for physical determinants.

- It is possible to rely on theoretical curves reached resulted from kinetic analysis in this research to assess skill performance level. 
- Proposed training program significantly improved physical variables under study (motor reaction speed - accuracy - fine motor reaction speed)

- Results illustrated the importance of motor reaction speed exercises and associate it with accuracy, as well as the importance for exercises similar to skill performance in improving Mawashi-geri lick's skill performance

\section{Recommendations:}

In light of research objectives, , within sample limits and research results; researcher recommend the following:

- Attention should be given to develop motor reaction speed and associate it with accuracy because of its importance in scoring points and winning matches in kumite.

- Using kinematic determinants derived from this research when developing an objective and quantitative tests to evaluate performance of the skill under study.

- It is recommended to take advantage of specific exercises within the proposed training program as an important and influential component in development of skills performance in karate training sessions within special and competition preparation periods.

- Attention should be given to select juniors in the light of biomechanics determinants and who are characterized by long legs and attention should be given to train juniors to reach for the highest possible distance when making kicks and necessity to ensure leg contact with the target without displacing it and calculate time for accessing target and repeat performance in order to achieve minimum possible time to access target.

- Interest should be given in using kinetic analysis to determine Egyptians karate players status and develop it through fine details that are difficult to observe with eyes.

- Attention should be given to the proposed exercises similar to skill performance $s$ which take the same motor path for karate skills performance so that the training process is continuous and based on analytical thought and selecting kinetic analysis as a scientific and objective evaluation method with high degree of accuracy.

- Further studies to be done in karate for other and different age stages to correlate physical and biomechanics determinants and identify its impact on technical determinants and take advantage of previous studies and researches and link it with current researches.

\section{References:}

1. Aboul-Ela Abdel Fattah(1998): Sports biology and athlete health, Arab Thought House, Cairo. (in Arabic language. (in Arabic Language)

2. Ahmed Mahmoud Ibrahim (1999): planning principles for educational and training programs for karate, Dar EIMaaref, Alexandria.(in Arabic Language)

3. Ahmed Mahmoud Ibrahim(1991): Development of some special physical abilities and its impact on performance level of punches and for karate juniors from 10-12 years, $\mathrm{PhD}$ Thesis, Faculty of Physical Education, Alexandria. (in Arabic Language) 
4. Jamal Mohammed Aladdin (1994): laboratory studies in sports movements biomechanics, Knowledge House, Alexendria (in Arabic language.

5. Sawsan Abdul Moneim(1997): Biomechanics in sports field, Knowledge House, Cairo.(in Arabic language)

6. Sherif Mohamed Al-Awadi(1989): An analysis of some attack waves for high levels karate players, unpublished Ph.D. thesis, Faculty of Physical Education, Minya. (in Arabic Language).

7. Sherif Mohamed Al Awadi, Omar Mohammed Labib (2004): attack rules, Kumite, Cairo. (in Arabic Language).

8. Talha Hossam El Din (1998): biomechanics and integration between theory and practice in sports field, second edition, unique and revised, book publishing house, Cairo. ( in Arabic language)

9. Essam Abdul-Khaliq(1991): sports training theories and applications - 6 ed., Knowledge House, Cairo. (in Arabic language)

10. Imad Abdel Fattah Alserssi(2001): Impact of training program in development of physical and skills characteristics for karate players, unpublished Ph.D. thesis, Faculty of Physical Education, Tanta University. (in Arabic language)

11. Imad Abdel Fattah Alserssi(2004): Training program to improve speed associated with accuracy in light of the electrophoresis analysis of back reverse roundhouse kick for karate players, published research - Scientific journal for Physical Education Sciences - Faculty of Physical Education, Tanta University. (in Arabic language)

12. Imad Abdel Fattah Alserssi (2011): kumite competitions law (translation - explanation Analysis) Egyptian Karate Federation. (in Arabic language)

13. Amr Mohamed Taha Hloich (2002): Program to improve productivity of long and short front straight punch long and short in light of its kinematic properties for karate players, Ph.D. thesis, unpublished, Faculty of Physical Education, Tanta University. (in Arabic Language)
14. Alaa Mohammed Taha Hloich, Imad Abdel Fattah Alserssi, Amr Mohamed Taha Hloich (2005): Kinematic characteristics of skill from abroad as some of muscle development determinants for karate players, published research, Journal of Menoufia University for Physical Education and Sports. (in Arabic Language)

15. Mohamed Ibrahim Shehata, Mohammed Gaber Briqa, Mohamed Bayoumi (1996): Multimedia in mechanical analysis, theories and applications journal, Faculty of Physical Education for Men, Alexandria University, No. XIV. (in Arabic Language)

16. Mohammed Gaber Briqa, Ahmed Mahmoud Ibrahim: Biomechanics analysis of front kick in karate, Journal of Physical Education, Faculty of Physical Education, University of Zagazig, Volume XVI. (in Arabic Language)

17. Mohamed Hassan Allawi (1990): Sports training science, Knowledge House, Cairo.(in Arabic Language)

18. Mohammad Hassan Allawi, Abul-Ela Abdel Fattah(1984): Sports training physiology Arab Thought House, Cairo. (in Arabic language)

19. Wageh Ahmed Shamandi (2002): Preparing Karate players for Championship, theory, practice, Dar ElKotb, Cairo. (in Arabic language)

20. Charles B., Ruth (1994): Concepts of physical Fitness, WMC Brown publishers, pullover, U.S.A.

21. Nakyoma,. M.,: Best Karate series fou, Kodansha, International Co., Tokyo, 1979.

22. Okazaki, T., \& Strcivic, M.,: he text book of modern kavat, kodansha, International 1rd, New York, 1984.

23. Oyama, M.,: Karate, Japan publication trading, co., Tokyo, 1986.

24. Vershahansky (1993): Karate techniques, Vnited stats of Amirca, 1993.

25. WWW.WKF.net.

26. WWW.Karate \& arab.com 


\section{Appendixes}

Appendix (1)

program exercises

\section{I - warm-up exercises.}

1-Running around Karate playground.

2-Lateral running around Karate stadium.

3-(Standing forward strike) forward strike exchanging legs.

4-(Standing waist stabled) jump back and forward exchanging feet on square of $1 \mathrm{~m} \times 1 \mathrm{~m}$.

5-(Standing front position) jump back and forward exchanging feet

6-Jump back and forward with both feet on mat squares

7-Running with exchange leg opened out to the right and left with ground squares.

\section{II - flexibility exercises and prolong:}

Used in intra-rest periods.

1- (Slant standing) exchange bent and extend neck muscles.

2- Exchange knee turning to both sides.

3- Exchange neck turnover left and right.

4- (Bend standing ) pressing down.

5- (Standing) exchange trunk bent for both sides.

6- (Forward strike) pressing down.

7- (Backward strike) pressing down.

8- (Open standing) feet apart to maximum extent.

9- (Long open seating) bent trunk down forward.

10- (Stand against wall ladder ) exchange swinging either legs high forward backward.

11- (Stand against wall ladder ) exchange swinging either legs Inner side high.

12- (Stand against wall ladder ) exchange swinging high backward.

\section{III - motor reaction speed exercises:}

All exercises with a coach signal (audio or visual).

1-(Horizontal kneeling) exchange knees rising.

1-(Horizontal Kneeling) exchange knees backward extending.
2-(Horizontal Kneeling) exchange rising knees aside then extending knees backward.

3-(Horizontal kneeling, based on one knee ) Exchange bend and extend knee without touching the ground.

4-(Stand against wall) to extend knee and fix it the largest possible time.

5-(Stand against wall) quickly rotate when heard coach's voice.

6-Repeat the above with Sandstone bag fixed on thigh bone, or tibia and Rubber.

\section{IV - Accuracy drills:}

1-Skill performance on racket $50 \times 50 \mathrm{~cm}$.

2-Skill performance on racket 40 x $50 \mathrm{~cm}$.

3 -Skill performance on racket $30 \times 30 \mathrm{~cm}$.

4-Skill performance on racket $20 \times 20 \mathrm{~cm}$.

5-Skill performance on racket $10 \times 10 \mathrm{~cm}$.

\section{V - Fine motor reaction speed exercises:}

Integration of motor reaction speed exercises motor with previous accuracy exercise i.e exercise from No. 19 to No. 37. 
